\title{
XMM-Newton observations of 4 luminous radio-quiet AGN, and the soft X-ray excess problem
}

\author{
F. D’Ammando ${ }^{1,2}$, S. Bianchi ${ }^{1}$, E. Jiménez-Bailón ${ }^{1,3,4}$, and G. Matt ${ }^{1}$ \\ 1 Dipartimento di Fisica, Università degli Studi Roma Tre, via della Vasca Navale 84, 00146 Roma, Italy \\ e-mail: filippo.dammando@iasf-roma.inaf.it \\ 2 Dipartimento di Fisica, Università di Roma Tor Vergata, via della Ricerca Scientifica 1, 00133 Roma, Italy \\ 3 LAEFF-INTA, Apdo. 50727, 28080-Madrid, Spain \\ 4 Universidad Nacional Autonoma de Mexico, Apartado Postal 70-264, 04510 Mexico, DF, Mexico
}

Received 17 September 2007 / Accepted 29 January 2008

\begin{abstract}
Context. The nature and origin of the soft X-ray excess in radio-quiet AGN is still an open issue. The interpretation in terms of thermal disc emission has been challanged by the discovery of the constancy of the effective temperature despite the wide range of Black Hole masses of the observed sources. Alternative models are reflection from ionized matter and absorption in a relativistically smeared wind.

Aims. We analyzed XMM-Newton observations of four luminous radio-quiet AGN with the aim of characterising their main properties and in particular the soft excess.

Methods. Different spectral models for the soft excess were tried: thermal disc emission, Comptonization, ionized reflection, relativistically smeared winds.

Results. Comptonization of thermal emission and smeared winds provide the best fits, but the other models also provide acceptable fits. All models, however, return parameters very similar from source to source, despite the large differences in luminosities, Black Hole masses and Eddington ratios. Moreover, the smeared wind model requires very large smearing velocities. The UV to X-ray fluxes ratios are very different, but do not correlate with any other parameter.

Conclusions. No fully satisfactory explanation for the soft X-ray excess is found. More informative data, such as observations in a broader energy band, are needed to make further progress.
\end{abstract}

Key words. galaxies: active - X-rays: galaxies - galaxies: quasars: individual: H0439-272 galaxies: quasars: individual: Fairall 1116 - galaxies: quasars: individual: PG0052+251

\section{Introduction}

While relatively low luminosity AGN are well studied in X-rays, the same can not be said for high luminosity ones, due to their paucity in the local Universe and therefore of their relative low fluxes. With the aim to populate the high $L-$ high flux portion of the parameter space, we proposed and obtained XMM-Newton observations of four sources, selected from the Grossan HEAO1 LMA catalogue to be bright $\left(F_{2-10}>10^{-11} \mathrm{erg} \mathrm{cm}^{-2} \mathrm{~s}^{-1}\right)$ and luminous $\left(L_{2-10}>10^{44} \mathrm{erg} \mathrm{s}^{-1}\right)$ : H0439-272, Ark 374, Fairall 1116 and PG0052+251 (see Table 1).

HEAO1 instruments scanned the whole sky from 1977 to 1979. The A-1 instrument Large Sky Survey (LASS, Wood et al. 1984) catalogue contains 322 sources brighter than 0.0036 LASS counts $/ \mathrm{s} / \mathrm{cm}^{2}\left(F_{2-10}>1.82 \times 10^{-11} \mathrm{erg} \mathrm{cm}^{-2} \mathrm{~s}^{-1}\right.$, assuming a power law spectrum with $\Gamma=1.7)$ and with $|b|>20$ (Grossan 1992). The A-1 instrument consisted of a set of proportional counters sensitive in the $2-20 \mathrm{keV}$ band, which observed the sky through passive collimators with a field of view of $1 \times$ 4 and $0.5 \times 1$ degrees. The precision with which the position of each X-ray source was determined was greatly improved by the simultaneous observation of the MC instrument (Modulation Collimator or A-3). The MC instrument produced a pattern of small, diamond-shaped error regions, only $1 \times 4$ arcmin each
(90\% confidence limit). The size of the final LASS/MC error box is therefore of $\sim 0.3 \mathrm{deg}^{2}$. Grossan and collaborators identified optical counterparts of 287 of the 322 objects in the LASS catalog (86\%). 96 of these objects are identified with AGN: the LASS-MC-AGN (LMA) sample (Grossan 1992), by correlation with catalogs of known sources and through optical photometry and spectroscopy performed by the MIT group.

Information on the four sources and on the related observations are summarized in Tables 1 and 2. Fluxes are derived from the best fit models (see Sect. 3). Unfortunately, the sources were all found at fluxes (and therefore luminosities) lower by at least a factor of 2 than in the HEAO-1 observations (not unexpectedly, as they all were just above the flux threshold). Fortunately, they were still bright enough to allow for a detailed spectral analysis.

In this paper, we report on the spectral analysis of these four sources, with particular emphasis on the soft excess.

A soft excess is present in the X-ray spectra of most Seyfert $1 \mathrm{~s}$ and radio-quiet quasars. First noted by Arnaud et al. (1985) and Singh et al. (1985), it is an emission below $\sim 1 \mathrm{keV}$ in excess of the extrapolation of the power law component dominating at higher energies.

The origin of the soft excess is still an open issue. In the past, it was often associated with the thermal emission of the 
Table 1. For each source: dates and exposure times of the observation, redshift and line-of-sight Galactic absorption.

\begin{tabular}{ccccc}
\hline \hline Source & Date & $\begin{array}{c}\text { EPIC-pn } \\
T(\mathrm{ks})\end{array}$ & $z$ & $\begin{array}{c}N_{\mathrm{Hg}} \\
10^{20} \mathrm{~cm}^{-2}\end{array}$ \\
\hline H0439-272 & $2005-08-13$ & 20 & 0.084 & 2.50 \\
Ark 374 & $2005-07-09$ & 25 & 0.063 & 2.21 \\
Fairall 1116 & $2005-08-28$ & 20 & 0.058 & 3.29 \\
PG 0052+251 & $2005-06-26$ & 20 & 0.155 & 4.81 \\
\hline
\end{tabular}

accretion disc. The large effective temperatures usually obtained (when compared to what is expected in a standard Shakura \& Sunyaev, 1973, accretion disc), were attributed to a slim accretion disc in which the temperature is raised by photon trapping (Abramowicz et al. 1998; Mineshige et al. 2000), in which case the accretion is super-Eddington, or to Comptonisation of EUV accretion disc photons (Porquet et al. 2004).

However, it has been shown recently that the disc temperature is remarkably constant, around $0.1-0.2 \mathrm{keV}$, regardless of the central object luminosity and mass (Gierlinski \& Done 2004; Crummy et al. 2006). This result is difficult to explain in any model of the soft excess related to disc continuum emission, as in any disc model the temperature is expected to vary with both the black hole mass and the accretion rate.

On the other hand, the constancy of the temperature may arise naturally if the soft excess is not related to thermal emission at all, but due to absorption/emission processes, the "temperature" actually measuring atomic transitions. There is a large increase in opacity in partially ionized material due to lines and edges of ionized O VII, O VIII and iron at $\sim 0.7 \mathrm{keV}$, which can lead to the apparent soft excess, provided that velocity smearing broadens and blurs the atomic features.

Two alternative scenarios have been proposed: a partially ionized, relativistically blurred reflection from the accretion disc (Crummy et al. 2006) and a velocity smeared, partially ionized absorption (Gierlinski \& Done 2004), the latter model further developed by Schurch \& Done (2006) by including the emission associated with the absorbing material and proposing an origin in a "failed wind".

In the reflection model, the partially ionized material is identified with the accretion disc, but to produce a strong soft excess the intrinsic continuum must be significantly suppressed, perhaps by disc fragmentation or by light-bending effects (Fabian et al. 2002, 2004, 2005; Miniutti \& Fabian 2004).

In the absorption model, the partially ionized material is thought to be a wind likely starting from the disc (Schurch \& Done 2006). If this is the case, the velocity structure has to be complex, giving rise to a substantial broadening to mask the sharp atomic features, which otherwise should have been observed in high resolution gratings observations. A problem with this interpretation, as noted by the same authors, is that such winds tend to be produced within $\sim 25^{\circ}$ of the equatorial plane. Most of type-1 AGN are expected to have lines of sight that would not intercept much of this material, but this is in conflict with the fact that most type-1 AGN show a soft excess. Moreover, the required large velocity smearing implies a massloss rate much larger than the accretion rate required to power the observed luminosity. Schurch \& Done (2006) then proposed a "failed wind" instead, which does not require a large mass-loss rate. In such a case, the central X-ray source is strong enough to overionize the wind removing the acceleration before the material reaches escape velocity, allowing the material to fall back on the disk.

\section{Observations and data reduction}

All the sources were observed by XMM-Newton with all the EPIC CCD cameras, the p-n (Strüder et al. 2001) and the two MOS (Turner et al. 2001) cameras, with the RGS (der Herder et al. 2001) and the OM (Mason et al. 2001). To reduce pileup to negligible values (about $0.1 \%$ in the p-n) we decided to adopt small window modes for all sources and all EPIC instruments, except for MOS1, for which we used a full window mode in order to check for possible confusion problems within the HEAO1 error box.

Data were reduced with the Science Analysis System (SAS) v.7.1.0 software package, using standard procedures; screening for intervals of flaring particle background in the EPIC data was done consistently with the choice of the extraction radii, in order to maximise the signal-to-noise ratio, similarly to Piconcelli et al. (2004).

We do not make use of MOS data in this paper because the MOS data are slightly piled-up, despite the small window mode adopted for MOS2, and the addition of the MOS in the spectral fits do not significantly increase the precision with which spectral parameters are determined. We do not make use of the RGS data either, because an inspection of their spectra shows they are featureless.

P-n spectra were rebinned in order to have at least 20 counts/bin and to oversample the energy resolution by a factor of about 3. Patterns 0 to 4 were included in the $p-n$ spectrum, whose count rate is lower than the maximum for $1 \%$ pile-up (see Table 3 of Ehle et al. 2005).

Five exposures with the Optical Monitor were also available, all with the UVM2 $(231 \mathrm{~nm})$ filter. The Observation Data Files (ODF) of the OM for each source were extracted and processed using the meta-task omichain of the XMM $S A S$, with standard procedures.

All spectra were analyzed using Xspec v.12.3.1 (Arnaud 1996). In the following, all energies are in the source rest frame, and errors correspond to the $90 \%$ confidence level for one interesting parameter $\left(\Delta \chi^{2}=2.71\right)$, unless otherwise stated.

Luminosities were calculated considering a flat $\Lambda \mathrm{CDM}$ cosmology with $\left(\Omega_{\mathrm{M}}, \Omega_{\Lambda}\right)=(0.3,0.7)$ and $H_{0}=70 \mathrm{~km} \mathrm{~s}^{-1} \mathrm{Mpc}^{-1}$ (Bennett et al. 2003). Metal abundances have been kept fixed to cosmic ones, according to Anders \& Grevesse (1989).

\section{Data analysis}

\subsection{Baseline fit model}

We started the analysis by fitting the p-n instrument only, in the $0.5-10 \mathrm{keV}$ energy range. A simple power law (absorbed by the Galactic column) gives unacceptable fits, with strong excess residuals at low energies (see Fig. 1). The inclusion of a neutral Compton reflection component (PEXRAV in Xspec) does not help much, unless very large and unrealistic values (given also the iron line equivalent widths, see below) for the relative normalizations are allowed. We added a further power law to reproduce the soft excess. We call this baseline model spectrum (BMS), which consists of two power laws, cold absorption from the Galactic hydrogen column density and a Gaussian line to reproduce the iron $\mathrm{K} \alpha$ fluorescent emission line. We fixed the rest frame energy of the line to $6.4 \mathrm{keV}$ and the width at $10^{-3} \mathrm{keV}$; the fits did not improve when these parameters were left free to vary (see below).

The fit of the BMS was very good for all sources (reduced $\chi^{2}$ of about 1), except for H0439-272, for which inspection of residuals suggested the inclusion of an absorption edge at $\sim 0.72 \mathrm{keV}$ 
Table 2. For each source: $0.5-2$ and 2-10 keV flux and luminosity; bolometric luminosity (derived adopting Marconi et al. (2004) bolometric corrections); Black Hole mass, Eddington ratio and $\mathrm{H}_{\beta} F W H M$. Black Hole masses are from: Shields et al. (2003) for Ark 374 (lower value) and Fairall 1116; from Vestergaard \& Peterson (2006) for Ark 374 (upper value) and PG 0052+251; and from the H $\beta$ FWHM reported in Grupe et al. (2004) for H0439-272, using the formula in Vestergaard \& Peterson (2006). Other values of the H $\beta$ FWHM are from Corbin 1991 (Ark 374), Grupe et al. 2004 (Fairall 1116) and Peterson et al. 2004 (PG 0052+251).

\begin{tabular}{|c|c|c|c|c|c|c|c|}
\hline Source & $\begin{array}{c}F_{0.5-2} \\
\left(10^{-12} \mathrm{erg} \mathrm{cm}^{-2} \mathrm{~s}^{-1}\right)\end{array}$ & $\begin{array}{c}F_{2-10} \\
\left(10^{-12} \mathrm{erg} \mathrm{cm}^{-2} \mathrm{~s}^{-1}\right)\end{array}$ & $\begin{array}{c}L_{2-10} \\
\left(10^{43} \mathrm{erg} \mathrm{s}^{-1}\right)\end{array}$ & $\begin{array}{c}L_{\mathrm{B}} \\
\left(10^{43} \mathrm{erg} \mathrm{s}^{-1}\right)\end{array}$ & $\begin{array}{c}M_{\mathrm{BH}} \\
\left(10^{8} M_{\odot}\right)\end{array}$ & $\bar{\lambda}$ & $\begin{array}{c}F W H M \mathrm{H}_{\beta} \\
\mathrm{km} \mathrm{s}^{-1}\end{array}$ \\
\hline H0439-272 & 4.54 & 5.74 & 10.0 & 34.0 & 0.36 & 0.73 & 2500 \\
\hline Ark 374 & 3.16 & 3.29 & 3.1 & 7.2 & $0.74-1.4$ & $0.04-0.075$ & 4200 \\
\hline PG $0052+251$ & 5.03 & 6.77 & 42.6 & 230 & $3.7-8.5$ & $0.21-0.48$ & 4165 \\
\hline
\end{tabular}

Table 3. Best fit parameters for the BMS: wabs (powerlaw+powerlaw+zgauss). In the case of H0439-272, an edge with $E_{\mathrm{th}}=0.72 \pm 0.02$ and $\tau=$ $0.27 \pm 0.05$ has been added.

\begin{tabular}{cccccc}
\hline \hline Object & $\Gamma_{1}$ & $\Gamma_{2}$ & $\begin{array}{c}E W_{\mathrm{Fe}} \\
(\mathrm{eV})\end{array}$ & $\chi_{\mathrm{r}}^{2} /$ d.o.f. & Null Hyp. Prob. \\
\hline H0439-272 & $1.54_{-0.37}^{+0.15}$ & $2.63_{-0.23}^{+0.33}$ & $54 \pm 36$ & $1.11 / 184$ & 0.15 \\
Ark 374 & $1.43_{-0.23}^{+0.26}$ & $2.72_{-0.16}^{+0.22}$ & $71_{-42}^{+50}$ & $0.97 / 167$ & 0.61 \\
Fairall 1116 & $1.56_{-0.23}^{+0.10}$ & $2.93_{-0.23}^{+0.17}$ & $113 \pm 41$ & $1.01 / 190$ & 0.44 \\
PG0052+251 & $1.25_{-0.20}^{+0.12}$ & $2.71_{-0.12}^{+0.14}$ & $<61$ & $0.92 / 192$ & 0.77 \\
\hline
\end{tabular}

(consistent with the $\mathrm{O}$ VII $\mathrm{K}$ edge) which indeed reduces the $\chi^{2}$ from 251 (186 d.o.f.) to 204 (184 d.o.f.). The best fit parameters for BMS (including the absorption edge for H0439-272) are summarized in Table 3.

\subsection{The iron line}

Two sources, Ark 374 and Fairall 1116, have a strong enough iron line to search for complexities in their profiles.

For Ark 374, leaving the energy and width of the line free to vary, a marginal improvement is found $\left(\Delta \chi^{2}=5.6\right)$. The best fit centroid energy and width are $6.48_{-0.07}^{+0.20} \mathrm{keV}$ and $0.18_{-0.08}^{+0.14} \mathrm{keV}$, respectively. Even less significant is the improvement for Fairall $1116\left(\Delta \chi^{2}=1.4\right)$. The best fit centroid energy and width are $6.43_{-0.04}^{+0.03} \mathrm{keV}$ and $<0.09 \mathrm{keV}$, respectively. For all sources, therefore, we can conclude that the iron line is consistent with being neutral and unresolved, even if a relativistic disc component cannot, of course, be excluded given the modest quality of the data.

Even considering the large error bars, the Equivalent Width of the iron line seems to diminishes with the luminosity (the Iwasawa-Taniguchi effect) and with the Eddington ratio, as found by Bianchi et al. (2007) in a much larger sample.

\subsection{The soft excess}

For all sources, a soft X-ray emission in excess of the extrapolation of the hard power law is clearly present (see Fig. 1). In the BMS model, the soft excess was reproduced by a simple power law. To investigate the nature of this component, we tried alternative models, representative of the different possible origins (for H0439-272 we also included the absorption edge as detailed in Sect. 3.1).

An important characteristic of the soft excess observed in previous studies is the constancy of its temperature when fitted with a thermal component (e.g. Gierlinki \& Done 2004), contrary to the expected $\propto M_{\mathrm{BH}}^{-\frac{1}{4}}$ relation, suggesting that the excess is related to atomic processes.

First, we checked whether the temperature is also constant in our small sample by substituting the soft power law with an accretion disc model consisting of multiple blackbody components (the DISKBB model in Xspec) to reproduce the soft excess (Table 4). The fits are acceptable but worse than with the 2 power law model. The temperature at the inner disc radius is very high and similar for all the sources (which have black hole masses different by up to an order of magnitude). The values are similar to those found by e.g. Piconcelli et al. (2005).

We then tried to reproduce the soft excess with a second Comptonization region (Table 5), using the simplest model available in Xspec, i.e. COMPST (Sunyaev \& Titarchuk 1980). This second region could be identified as a hot disc surface layer where electrons upscatter the photons emitted in deeper layers. The temperature of the electrons $k T_{\mathrm{e}}$ and the optical depth $\tau$ are again, and surprisingly, very similar for all the sources. (The energy of the injected photons is hidden in the normalization parameter; the spectral shape is determined by the electron temperature and the optical depth of the hot layer.) The quality of the fits is similar to those with the two power laws, and better than those with the multicolor disc model.

We then tried the two models that explain the soft excess in terms of atomic physics processes, namely the ionized disc reflection and the smeared absorption. To describe the reflection from the accretion disc we used the latest publicly available version of the code described by Ballantyne et al. (2001; see also Ross \& Fabian 2005; and Crummy et al. 2006), corrected by a convolution model for relativistic smearing (KDBLUR in Xspec). The reflection is characterized by the ionisation parameter of the reflecting matter, $\xi$, the photon index of the incident power law (which we assumed to be the same as the observed power law) and the reflection parameter. The parameters that characterize the relativistic smearing are the spin of the black hole, the inner and outer radii of the reflecting disc, the emissivity (parametrized as a power law) and the inclination angle. In 

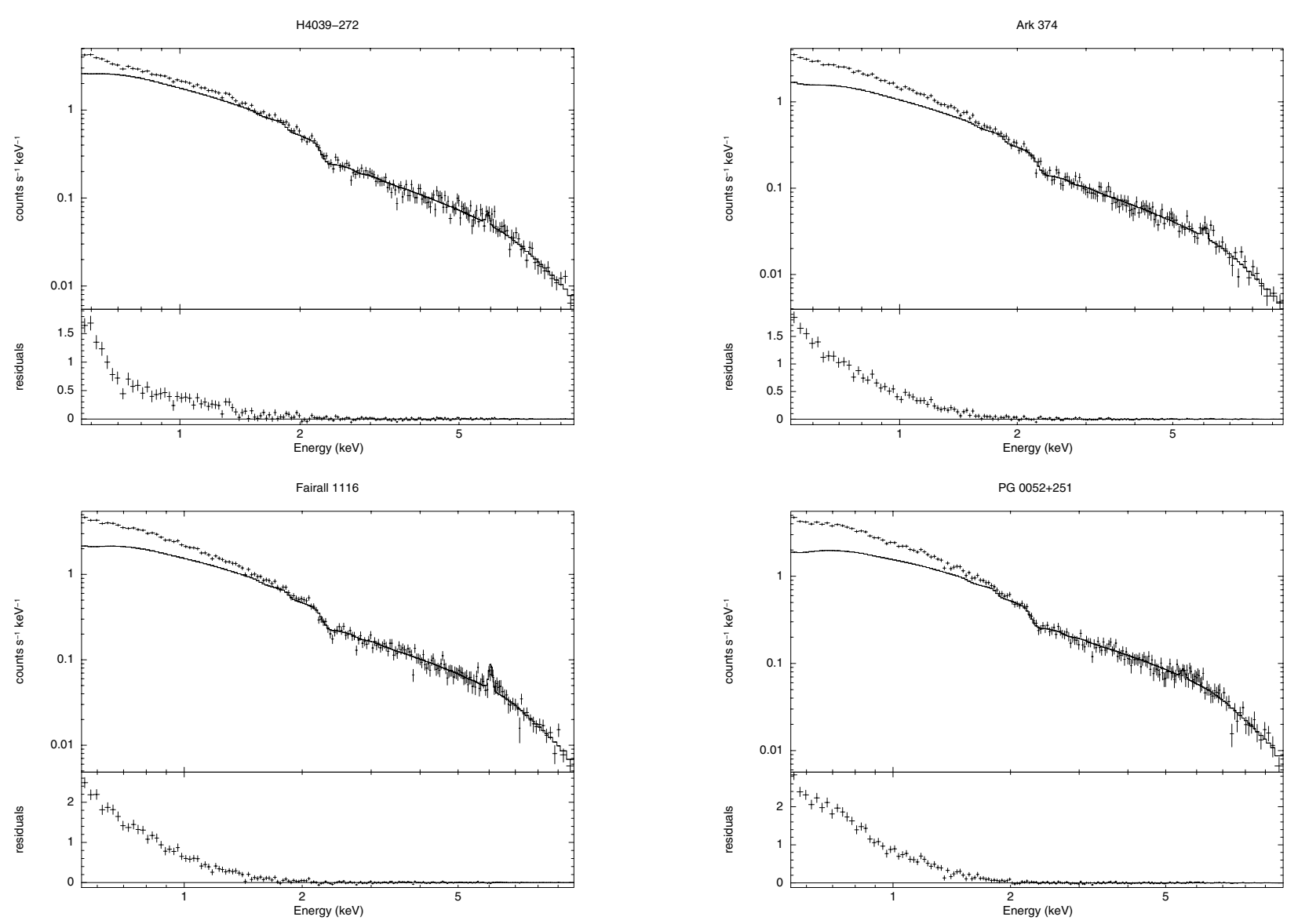

Fig. 1. Spectrum and best fit model (upper panel) and residuals (lower panel) of the 4 sources, when fitted with a single power law in the $2-10 \mathrm{keV}$ band, and then extrapolated to lower energies.

practice, and for simplicity, we assumed a maximally rotating black hole $(a=0.998)$ and fixed the emissivity index to 2 , the inclination angle to $30^{\circ}$, the inner and outer radii to 1.23 and 400 gravitational radii. The results are summarizeded in Table 6. In the table, the parameter "Fraction" indicates the ratio of the reflected to direct continuum at $15 \mathrm{keV}$, where the effects of ionization are small and the ratio is dominated by the geometry. For comparison, a neutral reflection component from a face-on slab with $R=1, \Gamma=2$ and $A_{\mathrm{Fe}}=1$ gives a fraction of 0.44 . The quality of the fit is worse than with the previous model. This may be (at least partly) due to our choice of keeping the disc parameter fixed (leaving the inner radius and the inclination angle free to vary, a significantly better fit, i.e. $\chi_{\mathrm{r}}^{2}=0.96 / 190$ d.o.f., is found only for PG0052+251, due to a much higher inclination, $86^{\circ}$ ) and the oversimplification of the model, e.g. a single ionization zone and a simple power law model for the emissivity law, which is much more complicated (see e.g. Martocchia et al. 2002) for the lamp-post geometry. The fraction of the reflection component ranges from 0.14 to 0.27 , i.e. between $1 / 3$ and almost $2 / 3$ of a whole disc seen face-on. The iron abundance is always about solar, while the ionization parameter spans a relatively narrow range of values. If the emissivity law is set to 3 , the quality of the fit is similar in H0439-272 and PG0052+251, and better (but still worse than with previous models) in Ark 374 and Fairall 1116 (see Table 6). The best fit parameters are all consistent within the errors with those obtained with an emissivity index of 2 , even if the values of the ionization parameter are always larger and the iron abundance smaller.
Table 4. Best fit parameters for the model: wabs (diskbb + powerlaw + zgauss).

\begin{tabular}{ccccc}
\hline \hline Object & $k T(\mathrm{keV})$ & $\Gamma$ & $\chi_{\mathrm{r}}^{2} /$ d.o.f. & Null Hyp. Prob. \\
\hline H0439-272 & $0.21_{-0.02}^{+0.02}$ & $1.90_{-0.04}^{+0.04}$ & $1.14 / 184$ & 0.09 \\
Ark 374 & $0.18_{-0.01}^{+0.01}$ & $1.97_{-0.05}^{+0.04}$ & $1.15 / 167$ & 0.09 \\
Fairall 1116 & $0.18_{-0.01}^{+0.81}$ & $1.89_{-0.04}^{+8.84}$ & $1.14 / 190$ & 0.09 \\
PG0052+251 & $0.19_{-0.01}^{+0.01}$ & $1.81_{-0.04}^{+0.04}$ & $1.12 / 192$ & 0.11 \\
\hline
\end{tabular}

Finally, we replaced the reflector with a relativistically smeared absorption (the SWIND1 model, developed by Gierlinski \& Done 2006; see Table 7), even if this model has been recently criticized by Schurch \& Done (2007a,b) as being oversimplified. The SWIND1 model has 3 parameters: the ionization, the density column and the Gaussian velocity smearing, $\sigma$. The fits are as good as the ones with two power laws. The column density and the ionization parameter of the absorbing wind are very similar, and the smearing terminal velocity is very high, close to $0.5 \mathrm{c}$. Fixing the latter parameter to a smaller value, $0.15 \mathrm{c}$, resulted in poorer, unacceptable fits $\left(\chi_{\mathrm{r}}^{2}>1.38\right.$, for the faintest source, Ark 374). Note that when a refined version of the model (not yet available for spectral fitting) is considered, Schurch \& Done (2007b) found that indeed extremely large, unrealistic smearing terminal velocities are required. 
Table 5. Best fit parameters for the model: wabs (compst + powerlaw + zgauss).

\begin{tabular}{cccccc}
\hline \hline Object & $k T(\mathrm{keV})$ & $\tau$ & $\Gamma$ & $\chi_{\mathrm{r}}^{2} /$ d.o.f. & Null Hyp. Prob. \\
\hline H0439-272 & $0.46_{-0.18}^{+5.74}$ & $20_{-18}^{+8}$ & $1.79_{-0.18}^{+0.08}$ & $1.10 / 185$ & 0.16 \\
Ark 374 & $0.41_{-0.11}^{+3.30}$ & $21_{-6}^{+6}$ & $1.80_{-0.16}^{+0.08}$ & $0.96 / 166$ & 0.62 \\
Fairall 1116 & $0.38_{-0.10}^{+0.32}$ & $22_{-5}^{+6}$ & $1.76_{-0.08}^{+0.08}$ & $1.00 / 189$ & 0.49 \\
PG0052+251 & $0.39_{-0.07}^{+0.26}$ & $22_{-6}^{+3}$ & $1.63_{-0.10}^{+0.05}$ & $0.91 / 191$ & 0.81 \\
\hline
\end{tabular}

Table 6. Best fit parameters for the model: wabs (kdblur(reflion) + powerlaw + zgauss). The upper four rows refer to the emissivity index fixed to 2 , the lower to 3 . See text for further details.

\begin{tabular}{ccccccc}
\hline \hline Object & $\xi$ & $\Gamma$ & Fraction & $A_{\mathrm{Fe}}$ & $\chi_{\mathrm{r}}^{2} /$ d.o.f. & Null Hyp. Prob. \\
\hline H0439-272 & $465_{-85}^{+120}$ & $1.96_{-0.02}^{+0.01}$ & 0.14 & $1.24_{-0.35}^{+0.48}$ & $1.15 / 185$ & 0.07 \\
Ark 374 & $550_{-174}^{+103}$ & $2.03_{-0.03}^{+0.04}$ & 0.22 & $1.01_{-0.16}^{+0.31}$ & $1.23 / 166$ & 0.02 \\
Fairall 1116 & $441_{-49}^{+76}$ & $1.94_{-0.03}^{+0.03}$ & 0.25 & $1.07_{-0.15}^{+0.23}$ & $1.15 / 189$ & 0.08 \\
PG0052+251 & $445_{-30}^{+75}$ & $1.90_{-0.03}^{+0.02}$ & 0.27 & $1.34_{-0.27}^{+0.34}$ & $1.14 / 191$ & 0.09 \\
\hline H0439-272 & $585_{-160}^{+340}$ & $1.96_{-0.03}^{+0.03}$ & 0.17 & $0.97_{-0.27}^{+0.64}$ & $1.15 / 185$ & 0.07 \\
Ark 374 & $728_{-193}^{+590}$ & $2.02_{-0.04}^{+0.03}$ & 0.25 & $0.86_{-0.19}^{+0.25}$ & $1.10 / 166$ & 0.18 \\
Fairall 1116 & $560_{-10}^{+130}$ & $1.93_{-0.03}^{+0.03}$ & 0.30 & $0.94_{-0.18}^{+0.26}$ & $1.05 / 189$ & 0.32 \\
PG0052+251 & $530_{-72}^{+84}$ & $1.91_{-0.03}^{+0.03}$ & 0.36 & $0.92_{-0.18}^{+0.34}$ & $1.16 / 191$ & 0.06 \\
\hline
\end{tabular}

Table 7. Best fit parameters for model: wabs (swind1 (powerlaw) + zgauss).

\begin{tabular}{ccccccc}
\hline \hline Object & $\begin{array}{c}\text { Column } \\
\times 10^{22} \mathrm{~cm}^{-2}\end{array}$ & $\log \xi$ & $\begin{array}{c}\sigma \\
(\mathrm{v} / \mathrm{c})\end{array}$ & $\Gamma$ & $\chi_{\mathrm{r}}^{2} /$ d.o.f. & Null Hyp. Prob. \\
\hline H0439-272 & $28.8_{-12.3}^{+21.2 *}$ & $3.41_{-0.17}^{+0.19}$ & $0.5^{*}$ & $2.04_{-0.04}^{+0.03}$ & $1.11 / 184$ & 0.15 \\
Ark 374 & $27.3_{-9.2}^{+11.0}$ & $3.32_{-0.12}^{+0.14}$ & $0.5^{*}$ & $2.13_{-0.03}^{+0.04}$ & $1.03 / 167$ & 0.38 \\
Fairall 1116 & $21.7_{-6.5}^{+7.7}$ & $3.21_{-0.15}^{+0.11}$ & $0.46_{-0.10}^{+0.04 *}$ & $2.09_{-0.03}^{+0.03}$ & $1.02 / 189$ & 0.39 \\
PG0052+251 & $35.4_{-8.4}^{+14.6 *}$ & $3.35_{-0.12}^{+0.10}$ & $0.46_{-0.12}^{+0.04 *}$ & $2.04_{-0.04}^{+0.03}$ & $0.94 / 191$ & 0.72 \\
\hline
\end{tabular}

* Parameter fixed, or error pegged to the boundary (see text for details).

Table 8. First column: UV fluxes, corrected for the extinction; second column: UV/X $(2-10 \mathrm{keV})$ flux ratio; third to sixth columns: the ratio between the extrapolated best fit models to $231 \mathrm{~nm}$ and the observed values.

\begin{tabular}{ccccccc}
\hline \hline Source & $\begin{array}{c}F_{\mathrm{UV}} \\
\mathrm{erg} \mathrm{cm}^{-2} \mathrm{~s}^{-1}\end{array}$ & $F_{\mathrm{UV}} / F_{\mathrm{X}}$ & $R_{\mathrm{BMS}}$ & $R_{\text {Diskbb }}$ & $R_{\text {Refl }}$ & $R_{\text {RelAbs }}$ \\
\hline H0439-272 & 5.29 & 0.92 & 11.2 & 0.36 & 0.17 & 1.1 \\
Ark 374 & 18.8 & 5.70 & 4.0 & 0.09 & 0.05 & 0.3 \\
Fairall 1116 & 0.90 & 0.17 & 300 & 1.7 & 1.9 & 9.2 \\
PG0052+251 & 35.7 & 5.27 & 3.6 & 0.03 & 0.04 & 0.2 \\
\hline
\end{tabular}

\subsection{The ultraviolet flux}

The UV fluxes of the Optical Monitor observations were obtained by simply converting the count rates with the method 1 described in the SAS documentation ${ }^{1}$. As we are only interested in comparing the UV and X-ray fluxes, rather than in performing detailed fitting of the Spectral Energy Distribution (SED), we did not perform a more sophisticated analysis. In Table 8 we report the UV fluxes of the sources, corrected for extinction, and the UV/X $(2-10 \mathrm{keV})$ flux ratio.

There are large differences between the UV/X fluxes ratios of the sources. There is no apparent relation with either the black

\footnotetext{
1 http://xmm.vilspa.esa.es/sas/7.0.0/watchout/ Evergreen_tips_and_tricks/UVflux.html
}

hole mass or the Eddington ratio. Given the rather constant values of the $0.5-2 / 2-10 \mathrm{keV}$ ratio and of the soft power law index, this suggests that the soft X-ray excess and the UV flux are not directly related to each other. In the table, the ratio between the extrapolation to $231 \mathrm{~nm}$ of the baseline, thermal disc, ionized disc reflection and relativistic absorption models to the observed values are also given. No model reproduces the UV flux for all sources. The power law (BMS model) always overpredicts the UV flux. The disc thermal emission strongly underpredicts the UV flux for Ark 374 and PG 0052+251. It is more difficult to judge the reflection and relativistic disc models, as the extrapolation to the UV must give a flux lower than the observed one, because these models do not include the disc thermal emission, which must contribute in the UV. Certainly, at 
least for Fairall 1116 both models fail (and the wind model for H0439-272 as well).

\section{Discussion and conclusions}

We have analyzed 4 moderately luminous AGN observed with the XMM-Newton satellite. The main results can be summarized as follows:

a) The hard X-ray emission is characterized by a power law with spectral indices that depends very much on the modeling of the soft excess. If the latter is modelled as a power law, the hard X-ray photon index is quite flat, $\Gamma$ ranging from 1.25 to 1.56. If, on the other hand, other parameterizations are chosen (some of them giving fits of comparable quality), much steeper $\Gamma$ are found, similar to those usually found in AGN (e.g. Porquet et al. 2004; Piconcelli et al. 2005; Bianchi et al., in prep.), except when using a smeared absorbing wind, when quite steep values, $\Gamma>2$, are found.

b) An iron line is also detected, even if in PG $0052+251$ only an upper limit is found. The error bars are very large, but the decrease of the best fit values of the $E W$ with both the luminosity and the the Eddington ratio is in agreement with what was found by Bianchi et al. (2007) in a much larger sample (the Iwawasa-Taniguchi effect).

c) No satisfactory modeling for the soft excess is found. A power law, besides resulting in suspiciously flat hard power laws, has no obvious physical meaning, and it will not be discussed further. We note that the Comptonization model (see below) is a power law (and indeed the fits are statistically comparable) with a cutoff (which results in a steeper hard power law).

A multicolor thermal disc emission, apart from providing significantly worse fits, results in very high temperatures, almost constant despite the factor $\sim 10$ differences in black hole masses, which should result in a factor $\sim 2$ differences in $k T$. A Comptonization model provides both good fits and "normal" hard power law indices, but the temperature and the optical depth of the Comptonizing electron are suspiciously the same for all objects, with no obvious reasons for such a constancy.

A (relativistically blurred) ionized reflection model provides poor fits (which may be attributed to the oversimplifications of the model, such as a single ionization zone while a centrally illuminated disc obviously has a strong radial dependence on $\xi$, e.g. Matt et al. 1993). Moreover, the range of ionization parameters spanned by the four sources is rather small, even if this may be due to the fact that the zone of the disc with those ionization parameters are the ones contributing most to the soft excess (smaller ionizations resulting in a smaller albedo, higher ionizations in a lower energy dependence of the albedo).

Finally, the relativistically smeared absorbing wind model provides good fits to the data, but the three model parameters (column density, ionization parameter and smearing velocity) are almost constant among the sources. While there may be an explanation for the constancy of the column and the ionization parameter (see discussion in Middleton et al. 2006), the constancy of the smearing velocity and, above all, its high value (about $0.5 c$ ) are difficult to explain. Outflowing winds with velocities as high as $0.1-0.2 c$ have been observed in a few high accretion rate sources (e.g. Pounds et al. 2003). Fixing the smearing velocity to 0.15 , however, results in unacceptable fits. Schurch \& Done (2007b), using a more

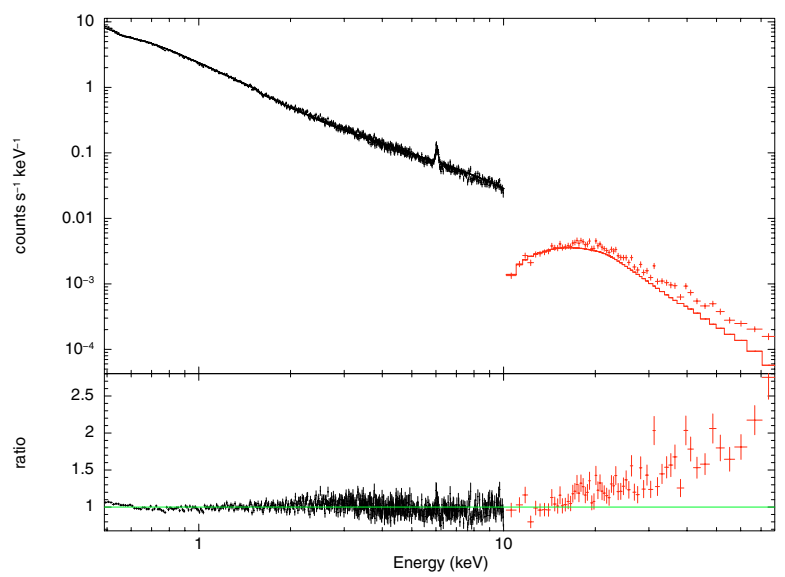

Fig. 2. A $100 \mathrm{ks}$ Simbol-X simulations of the baseline model spectrum of Fairall 1116 (upper panel), and the data/model ratio when the spectrum is fitted with the smeared absorption model (lower panel).

refined version of the model (not availaible for spectral fitting), found that indeed extremely large, unrealistic velocities are required to explain the soft X-ray excesses in AGN.

Thus, none of the abovementioned models provides a satisfactory description of the data, even if none of them can be completely ruled out. The constancy of the parameters we found for all models is clearly telling us that some characteristic energy is involved. Future, high sensitivity broad band measurements like those provided by e.g. Simbol-X (Ferrando et al. 2006) will hopefully tell us which, if any, of these model is tenable (Matt 2007; Ponti et al. 2007). As an example, in Fig. 2 the Simbol-X simulation of the baseline model for Fairall 1116 is shown, as well as the data/model ratio after fitting with the smeared absorption model.

d) The UV/X ratio is very different from source to source by more than a factor 30 - and does not correlate with any other parameter. The soft-to-hard X-ray ratio is instead basically constant, suggesting that the UV and soft X-rays are not physically related - evidence against a thermal disc origin for the soft X-rays.

Acknowledgements. We thank the anonymous referee for valuable comments which helped us improve the clarity of the paper. This paper is based on observations obtained with XMM-Newton, an ESA science mission with instruments and contributions directly funded by ESA Member States and the USA (NASA). G.M., S.B. and E.J.B. acknowledge financial support from ASI under grant $\mathrm{I} / 023 / 05 / 0$.

\section{References}

Abramowicz, M. A., Czerny, B., Lasota, J. P., \& Szusziewicz, E. 1988, ApJ, 322, 646

Anders, E., \& Grevesse, N. 1989, Geo. Cosm. Acta, 53, 197

Arnaud, K. A. 1996, in Astronomical Data Analysis Software and Systems V., Astron. Soc. Pac., ed. G. H. Jacoby, \& J. Barnes, San Francisco, ASP Conf. Ser., 101, 17

Arnaud, K. A., Branduardi-Raymont, G., Culhane, J. L., et al. 1985, MNRAS, 217,105

Ballantyne, D. R., Iwasawa, K., \& Fabian, A. C. 2001, MNRAS, 323, 506

Bennett, C. L., Hill, R. S., Hinshaw, G., et al. 2003, ApJS, 148, 97

Bianchi, S., Guainazzi, M., Matt, G., \& Fonseca Bonilla, N. 2007, A\&A, 467, L19

Crummy, J., Fabian, A. C., Gallo, L., \& Ross, R. R. 2006, MNRAS, 365, 1067

Corbin, M. R. 1991, ApJ, 371, L51 
den Herder, J. W., Brinkman, A. C., Kahn, S. M., et al. 2001, A\&A, 365, L7

Ehle, M., Breitfellner, M., Gonzalez-Riestra, R., et al. 2005, XMM-Newton Users' Handbook, http://xmm.vilspa.esa.es/external/xmm_user_ support/documentation/uhb_2.1/

Fabian, A. C., Ballantyne, D. R., Merloni, A., et al. 2002, MNRAS, 331, L35

Fabian, A. C., Miniutti, G., Gallo, L., et al. 2004, MNRAS, 353, 1071

Fabian, A. C., Miniutti, G., Iwasawa, K., \& Ross, R. R. 2005, MNRAS, 361, 795 Ferrando, P., et al. 2006, in Space Telescopes and Instrumentation II: Ultraviolet to Gamma Ray, ed. M. J. L. Turner, \& G. Hasinger, Proc. SPIE, 6266, 62660 Gierlinski, M., \& Done, C. 2004, MNRAS, 349, L7

Gierlinski, M., \& Done, C. 2006, MNRAS, L64

Grossan, B. A. 1992, MIT Ph.D. Thesis

Grupe, D., Wills, B. J., Leighly, K. M., \& Meusinger, H. 2004, AJ, 127, 156

Marconi, A., Risaliti, G., Gilli, R., et al. 2004, MNRAS, 351, 169

Mason, K. O., Breeveld, A., Much, R., et al. 2001, A\&A, 365, L36

Martocchia, A., Matt, G., \& Karas, V. 2002, A\&A, 383, L23

Matt, G. 2007, in Simbol-X: the hard X-ray universe in focus, Mem. S. A. It., in press

Matt, G., Fabian, A. C., \& Ross, R. R. 1993, MNRAS, 262, 179

Mineshige, S., Kawaguchi, T., Takauchi, M., \& Hayashide, K. 2000, PASJ, 52, 499
Miniutti, G., \& Fabian, A. C. 2004, MNRAS, 349, 1435

Middleton, M., Done, C., \& Gierlinski, M. 2007, MNRAS, in press

Peterson, B. M., Ferrarese, L., Gilbert, K. M., et al. 2004, ApJ, 613, 682

Piconcelli, E., Jimenéz-Bailón, E., Guainazzi, M., et al. 2004, MNRAS, 351, 161

Piconcelli, E., Jimenéz-Bailón, E., Guainazzi, M., et al. 2005, A\&A, 432, 15

Ponti, G., et al. 2007, in Simbol-X: the hard X-ray universe in focus, Mem. S. A. It., in press

Porquet, D., Reeves, J. N., O’Brien, P., \& Brinkmann, W. 2004, A\&A, 422, 85

Pounds, K. A., Reeves, J. N., King, A. R., et al. 2003, MNRAS, 345, 705

Ross, R. R., \& Fabian, A. C. 2005, MNRAS, 358, 211

Schurch, N. J., \& Done, C. 2006, MNRAS, 371, 81

Schurch, N. J., \& Done, C. 2007a, MNRAS, 381, 1483

Schurch, N. J., \& Done, C. 2007b, MNRAS, in press

Shakura, N. I., \& Sunyaev, R. A. 1973, A\&A, 24, 337

Shields, G. A., Gebhardt, K., Salviander, S., et al. 2003, ApJ, 583, 124

Singh, K. P., Garmire, G. P., \& Nousek, J. 1985, ApJ, 297, 633

Strüder, L., Briel, U., Dennerl, K., et al. 2001, A\&A, 365, L18

Sunyaev, R. A., \& Titarchuk, L. G. 1980, A\&A, 86, 121

Turner, M. J. L., Abbey, A., Arnaud, M., et al. 2001, A\&A, 365, L27

Vestergaard, M., \& Peterson, B. M. 2006, ApJ, 641, 689

Wood, K. S., Meekins, J. F., Yentis, D. J., et al. 1984, ApJS, 56, 507 